\title{
On a Stević-Sharma operator from Hardy spaces to the logarithmic Bloch spaces
}

Yongmin Liu ${ }^{1}$ and Yanyan Yu ${ }^{2^{*}}$

${ }^{\text {*Correspondence: }}$

yuyanyan@xzit.edu.cn

${ }^{2}$ School of Mathematics and Physics Science, Xuzhou Institute of

Technology, Xuzhou, 221000, China Full list of author information is

available at the end of the article

\section{Abstract}

Let $H(\mathbb{D})$ denote the space of all analytic functions on the unit disc $\mathbb{D}$ of the complex plane $\mathbb{C}, \psi_{1}, \psi_{2} \in H(\mathbb{D})$, and $\varphi$ be an analytic self-map of $\mathbb{D}$. In this paper, we characterize the boundedness and compactness of a Stević-Sharma operator $T_{\psi_{1}, \psi_{2}, \varphi}$ from Hardy spaces $H^{p}$ (with $1 \leq p<\infty$ ) to the logarithmic Bloch spaces $\mathcal{B}_{\text {log }}$.

MSC: Primary 47B38; secondary 47B33; 46E15; 30H10

Keywords: Hardy space; logarithmic Bloch space; multiplication operator; composition operator; differentiation operator

\section{Introduction}

We begin with a brief review of relevant concepts and results in one complex variable. Let $\mathbb{D}=\{z \in \mathbb{C}:|z|<1\}$ be the open unit disc in the complex plane $\mathbb{C}, H(\mathbb{D})$ the class of all analytic functions on the unit disc.

For $0 \leq r<1, f \in H(\mathbb{D})$, we set

$$
\begin{aligned}
& M_{p}(f, r)=\left(\frac{1}{2 \pi} \int_{0}^{2 \pi}\left|f\left(r e^{i \theta}\right)\right|^{p} \mathrm{~d} \theta\right)^{1 / p}, \quad 0<p<\infty, \\
& M_{\infty}(f, r)=\max _{0 \leq \theta \leq 2 \pi}\left|f\left(r e^{i \theta}\right)\right| .
\end{aligned}
$$

For $0<p<\infty$, the classical Hardy space $H^{p}$ is the space of all analytic functions $f$ on the unit disk $\mathbb{D}$ such that

$$
\|f\|_{H^{p}}=\sup _{0<r<1} M_{p}(f, r)<\infty
$$

It is well known that with the norm (1.1) the $H^{p}$ space is a Banach space if $1 \leq p<\infty$, for $0<p<1, H^{p}$ space is a nonlocally convex topological vector space, and $d(f, g)=\|f-g\|_{H^{p}}^{p}$ is a complete metric for it. Let $H^{\infty}$ denote the space of all $f \in H(\mathbb{D})$ for which $\|f\|_{\infty}=$ $\sup _{z \in \mathbb{D}}|f(z)|<\infty$. For more information about the $H^{p}$ space, one may see, for example, $[1,2]$.

The logarithmic Bloch space is defined as follows [3, 4]:

$$
\mathcal{B}_{\log }=\left\{f \in H(\mathbb{D}):\|f\|=\sup _{z \in \mathbb{D}}\left(1-|z|^{2}\right) \log \frac{2}{1-|z|}\left|f^{\prime}(z)\right|<\infty\right\} .
$$


The space $\mathcal{B}_{\log }$ is a Banach space under the norm $\|f\|_{\mathcal{B}_{\log }}=|f(0)|+\|f\|$. Let $\mathcal{B}_{\log , 0}$ denote the subspace of $\mathcal{B}_{\log }$ consisting of those $f \in \mathcal{B}_{\log }$ such that

$$
\lim _{|z| \rightarrow 1}\left(1-|z|^{2}\right) \log \frac{2}{1-|z|}\left|f^{\prime}(z)\right|=0 .
$$

It is obvious that there are unbounded $\mathcal{B}_{\log }$ functions. For example, consider the function $f(z)=\log \log \frac{e}{1-z}$. There are also bounded function that they do not belong to $\mathcal{B}_{\log }$. Particularly consider the inner function $S(z)=e^{\gamma \frac{z+\eta}{z-\eta}}$, where $\gamma \in(0,1)$ and $\eta \in \partial \mathbb{D}$. Then

$$
\left|S^{\prime}(z)\right|\left(1-|z|^{2}\right) \log \frac{2}{1-|z|}=2 \gamma e^{-\gamma \frac{1-|z|^{2}}{|z-\eta|^{2}}} e^{\frac{2 \gamma \operatorname{Re}(z \eta \eta)}{|z-\eta|^{2}}} \frac{1-|z|^{2}}{|z-\eta|^{2}} \log \frac{2}{1-|z|^{2}} .
$$

If let $z \rightarrow \eta$ on the horocycle $\frac{1-|z|^{2}}{|z-\eta|^{2}}=c$, we get $S \notin \mathcal{B}_{\log }$. Actually this is not the only bounded function which does not belong to $\mathcal{B}_{\text {log }}$. Consider an interpolating Blaschke product. That is a product $\prod_{k \geq 1} \frac{z_{k}-z}{1-\bar{z}_{k} z}:=B(z)$, where $\left\{z_{k}\right\} \subset \mathbb{D}$ such that $\sum_{k \geq 1}\left(1-\left|z_{k}\right|\right)<\infty$ and satisfies the following property. There exists $\delta \in(0,1)$ such that $\prod_{k \neq j}\left|\frac{z_{k}-z_{j}}{1-\bar{z}_{k} z_{j}}\right| \geq \delta$, for each $j \in\{1,2, \ldots\}$. Then we observe that for each $j$,

$$
\left|B^{\prime}\left(z_{j}\right)\right|\left(1-\left|z_{j}\right|^{2}\right) \log \frac{2}{1-\left|z_{j}\right|}=\prod_{k \neq j}\left|\frac{z_{k}-z_{j}}{1-\bar{z}_{k} z_{j}}\right| \log \frac{2}{1-\left|z_{j}\right|} \geq \delta \log \frac{2}{1-\left|z_{j}\right|} .
$$

So, it is obvious that the interpolating Blaschke products do not belong to $\mathcal{B}_{\log }$ but to $H^{\infty}$ [5, Theorem 15.21]. It is easily proved that for $0<\alpha<1, \mathcal{B}_{0}^{\alpha} \varsubsetneqq \mathcal{B}_{\log , 0} \varsubsetneqq \mathcal{B}_{0}$ and $\mathcal{B}^{\alpha} \varsubsetneqq \mathcal{B}_{\log } \varsubsetneqq$ $\mathcal{B}$, here $\mathcal{B}_{0}^{\alpha}$ is the little $\alpha$-Bloch space and $\mathcal{B}^{\alpha}$ is the $\alpha$-Bloch space.

The space $\mathcal{B}_{\log }$ arises in connection to the study of certain operators with symbol. Arazy in [6] proved that the multiplication operator $M_{\psi}$ is bounded on the Bloch space if and only if $\psi \in H^{\infty} \cap \mathcal{B}_{\text {log. }}$. In [7], Brown and Shields extended this result to the little Bloch space. Li and Stević in [8, Theorem 2.5] proved that $I_{g}: \mathcal{Z} \rightarrow \mathcal{Z}$ is bounded if and only if $g \in H^{\infty} \cap \mathcal{B}_{\log }$, here $I_{g} f(z)=\int_{0}^{z} f^{\prime}(\xi) g(\xi) \mathrm{d} \xi$.

The space $\mathcal{B}_{\log }$ appeared in the study of the boundedness of the Hankel operators on the Bergman space. Attele in [9] proved that for $f \in L_{a}^{2}(\mathbb{D})$, the Hankel operator $H_{f}: L_{a}^{1}(\mathbb{D}) \rightarrow$ $L^{1}(\mathbb{D})$ is bounded if and only if $\|f\|_{\mathcal{B}_{\log }}<\infty$, thus giving one reason, and not the only reason, why $\log$-Bloch-type spaces are of interest. Ye in [3] proved that $\mathcal{B}_{\log , 0}$ is a closed subspace of $\mathcal{B}_{\text {log. }}$. For some recent papers on some operators on $\mathcal{B}_{\text {log }}$, see, for example, [4, 10-18].

The composition, multiplication, and differentiation operator on $H(\mathbb{D})$ are defined as follows:

$$
\begin{aligned}
& \left(C_{\varphi} f\right)(z)=(f \circ \varphi)(z)=f(\varphi(z)), \quad z \in \mathbb{D} ; \\
& \left(M_{\psi} f\right)(z)=\psi(z) f(z), \quad z \in \mathbb{D} ; \\
& D f(z)=f^{\prime}(z), \quad z \in \mathbb{D} .
\end{aligned}
$$

The differentiation operator is typically unbounded on many analytic function spaces. For $\psi_{1}, \psi_{2} \in H(\mathbb{D})$, let

$$
T_{\psi_{1}, \psi_{2}, \varphi} f(z)=\psi_{1}(z) f(\varphi(z))+\psi_{2}(z) f^{\prime}(\varphi(z)), \quad f \in H(\mathbb{D})
$$


The operator $T_{\psi_{1}, \psi_{2}, \varphi}$ was studied by Stević and co-workers for the first time in [19, 20], also see [21]. This operator is related to the various products of multiplication, composition, and differentiation operators. It is clear that all products of composition, multiplication, and differentiation operator in the following six ways can be obtained from the operator $T_{\psi_{1}, \psi_{2}, \varphi}$ by fixing $\psi_{1}, \psi_{2}$. More specifically we have

$$
\begin{array}{ll}
M_{\psi} C_{\varphi} D=T_{0, \psi, \varphi} ; & M_{\psi} D C_{\varphi}=T_{0, \psi \varphi^{\prime}, \varphi} ; \quad C_{\varphi} M_{\psi} D=T_{0, \psi \circ \varphi, \varphi} ; \\
D M_{\psi} C_{\varphi}=T_{\psi^{\prime}, \psi \varphi, \varphi} ; & C_{\varphi} D M_{\psi}=T_{\psi^{\prime} \circ \varphi, \psi \varphi, \varphi} ; \quad D C_{\varphi} M_{\psi}=T_{\psi^{\prime} \circ \varphi \varphi^{\prime},(\psi \circ \varphi) \varphi^{\prime}, \varphi} .
\end{array}
$$

Product-type operators on some spaces of analytic functions on the unit disk have been the object of study for several recent years (see, for example, [22-38] and also related references therein). Ohno in [39] devoted most of the paper to finding necessary and sufficient conditions for $C_{\varphi} D$ to be bounded as well as for $C_{\varphi} D$ to be compact on the Hardy space $H^{2}$. The operator $D C_{\varphi}$ was studied for the first time in [40], where the boundedness and compactness of $D C_{\varphi}$ between Bergman and Hardy spaces are investigated. Li and Stević in $[23,27,28,41]$ studied the boundedness and compactness of the operator $D C_{\varphi}$ between Bloch-type spaces, weighted Bergman spaces $A_{\alpha}^{p}$ and $\mathcal{B}^{\alpha}$, mixed-norm space and $\mathcal{B}^{\alpha}$ as well as the space of bounded analytic functions and the Bloch-type space. Liu and Yu in [31] studied the boundedness and compactness of the operator $D C_{\varphi}$ from $H^{\infty}$ and Bloch spaces to Zygmund spaces. Yang in [42] studied the same problems for operators $C_{\varphi} D$ and $D C_{\varphi}$ from $Q_{K}(p, q)$ space to $\mathcal{B}_{\mu}$ and $\mathcal{B}_{\mu, 0}$. Stević in [35] studied the boundedness and compactness of the products of differentiation and multiplication operators $D M_{\psi}$ from mixednorm spaces to weighted-type spaces. Liu and $\mathrm{Yu}$ in [30] studied the operators $D M_{\psi}$ from $H_{\mu}^{\infty}$ to Zygmund spaces. Yu and Liu in [43] investigated the same problems for operators $D M_{\psi}$ from mixed-norm spaces to Bloch-type spaces. Zhu in [44] completely characterized the boundedness and compactness of linear operators which are obtained by taking products of differentiation, composition and multiplication operators, and which act from Bergman-type spaces to Bers spaces. Kumar and Singh in [45] investigated the same problem for operators $D C_{\varphi} M_{\psi}$ acting on $A_{\alpha}^{p}$ and used the Carleson-type conditions. They also found the essential norm estimates of $M_{\psi} D C_{\varphi}$ in the spirit of the work by Čuckovič and Zhao in [46]. Liang and Zhou in [47] investigated boundedness and compactness of the operators $C_{\varphi} D^{m}$ between $\mathcal{B}^{\alpha}$ and $\mathcal{B}^{\beta}$ and formulas for the essential norms were derived. Liang and Zhou in [48] found a new estimate of essential norm of composition followed by differentiation between Bloch-type spaces. Ye in [49] estimated the norm and the essential norm of composition followed by differentiation from logarithmic Bloch spaces to $H^{\infty}$. Hyvärinen and Nieminen in [50] investigated the behavior of $D u C_{\varphi}: \mathcal{B}^{\alpha} \rightarrow \mathcal{B}^{\beta}$, that is, the product of a weighted composition operator $u C_{\varphi}$ and the differentiation operator $D$, between Bloch-type spaces with standard weights. Further information on some related product-type operators on spaces of holomorphic functions on the unit ball are treated, for example, in [51-62].

Inspired by the above results, the purpose of the paper is to study the boundedness and compactness of the operator $T_{\psi_{1}, \psi_{2}, \varphi}$ from Hardy spaces $H^{p}$ (with $1 \leq p<\infty$ ) to the logarithmic Bloch spaces $\mathcal{B}_{\log }$. Throughout the paper, the letter $C$ denotes a positive constant which may vary at each occurrence, but it is independent of the essential variables.

The paper is organized as follows. Section 2 contains lemmas needed to prove Theorem 3.1, Corollary 3.2, Corollary 3.3, Theorem 3.4, Corollary 3.5, Corollary 3.6, Theo- 
rem 4.1, Corollary 4.2, Corollary 4.3, Theorem 4.4, Corollary 4.5, and Corollary 4.6. Section 3 considers the boundedness of the operator $T_{\psi_{1}, \psi_{2}, \varphi}: H^{p} \rightarrow \mathcal{B}_{\log }\left(\mathcal{B}_{\log , 0}\right)$. Section 4 considers the compactness of the operator $T_{\psi_{1}, \psi_{2}, \varphi}: H^{p} \rightarrow \mathcal{B}_{\log }\left(\mathcal{B}_{\log , 0}\right)$.

\section{Auxiliary results}

For a better understanding, in this section we list up the following four auxiliary results that are needed to prove our main results. The following lemma is folklore (see, e.g., [1, 2]).

Lemma 2.1 Assume that $p \in(0, \infty)$ and $f \in H^{p}$. Then for each $n \in \mathbb{N}_{0}$, there is a positive constant $C$ independent of $f$ such that

$$
\left|f^{(n)}(z)\right| \leq \frac{C\|f\|_{H^{p}}}{\left(1-|z|^{2}\right)^{n+1 / p}}, \quad z \in \mathbb{D}
$$

The following lemma in [1, p.65] plays an important role in characterizing the boundedness and the compactness of the operators under consideration in this paper.

Lemma 2.2 Assume that $p>1$, Then there is a positive constant $C(p)$ independent of $f$ such that

$$
\int_{0}^{2 \pi} \frac{\mathrm{d} \theta}{|1-z|^{p}} \leq \frac{C(p)}{\left(1-|z|^{2}\right)^{p-1}}, \quad z \in \mathbb{D} .
$$

The following criterion for the compactness follows by standard arguments (see, e.g., the proofs of the corresponding lemmas in [63, Proposition 3.11] or [64, Lemma 2.10]). The details will not be pursued here.

Lemma 2.3 Let $\psi_{1}, \psi_{2} \in H(\mathbb{D}), \varphi$ denotes an analytic self-map of $\mathbb{D}$. Then $T_{\psi_{1}, \psi_{2}, \varphi}: H^{p} \rightarrow$ $\mathcal{B}_{\log }$ is compact if and only if $T_{\psi_{1}, \psi_{2}, \varphi}: H^{p} \rightarrow \mathcal{B}_{\log }$ is bounded and for every bounded sequence $\left\{f_{n}\right\}$ in $H^{p}$ which converges to zero uniformly on compact subsets of $\mathbb{D}$ as $n \rightarrow \infty$, we have $\left\|T_{\psi_{1}, \psi_{2}, \varphi} f_{n}\right\|_{\mathcal{B}_{\log }} \rightarrow 0$ as $n \rightarrow \infty$.

The following lemma can be proved similar to Lemma 1 in [65] (see, also [66]). The details are omitted.

Lemma 2.4 $A$ closed set $K$ in $\mathcal{B}_{\log , 0}$ is compact if and only if it is bounded and satisfies

$$
\lim _{|z| \rightarrow 1} \sup _{f \in K}\left(1-|z|^{2}\right) \log \frac{2}{1-|z|}\left|f^{\prime}(z)\right|=0
$$

3 The boundedness of the operator $T_{\psi_{1}, \psi_{2}, \varphi}: H^{p} \rightarrow \mathcal{B}_{\log }\left(\mathcal{B}_{\log , 0}\right)$

First we consider the boundedness of the operator $T_{\psi_{1}, \psi_{2}, \varphi}: H^{p} \rightarrow \mathcal{B}_{\text {log }}$.

Theorem 3.1 Let $\psi_{1}, \psi_{2} \in H(\mathbb{D}), \varphi$ denote an analytic self-map of $\mathbb{D}$. Then the following statements are equivalent.

(a) $T_{\psi_{1}, \psi_{2}, \varphi}: H^{p} \rightarrow \mathcal{B}_{\log }$ is bounded;

(b)

$$
\sup _{z \in \mathbb{D}} \frac{\left(1-|z|^{2}\right) \log \frac{2}{1-|z|}\left|\psi_{1}^{\prime}(z)\right|}{\left(1-|\varphi(z)|^{2}\right)^{1 / p}}<\infty
$$




$$
\sup _{z \in \mathbb{D}} \frac{\left(1-|z|^{2}\right) \log \frac{2}{1-|z|}\left|\psi_{1}(z) \varphi^{\prime}(z)+\psi_{2}^{\prime}(z)\right|}{\left(1-|\varphi(z)|^{2}\right)^{1+1 / p}}<\infty
$$

and

$$
\sup _{z \in \mathbb{D}} \frac{\left(1-|z|^{2}\right) \log \frac{2}{1-|z|}\left|\psi_{2}(z) \varphi^{\prime}(z)\right|}{\left(1-|\varphi(z)|^{2}\right)^{2+1 / p}}<\infty .
$$

Proof (b) $\Rightarrow$ (a). First assume that (3.1), (3.2), and (3.3) hold. Then for every $z \in \mathbb{D}, f \in H^{p}$, by Lemmas 2.1 and 2.2 , we have

$$
\begin{aligned}
(1- & \left.|z|^{2}\right) \log \frac{2}{1-|z|}\left|\left(T_{\psi_{1}, \psi_{2}, \varphi} f\right)^{\prime}(z)\right| \\
= & \left(1-|z|^{2}\right) \log \frac{2}{1-|z|} \mid \psi_{1}^{\prime}(z) f(\varphi(z)) \\
& +\left(\psi_{1}(z) \varphi^{\prime}(z)+\psi_{2}^{\prime}(z)\right) f^{\prime}(\varphi(z))+\psi_{2}(z) \varphi^{\prime}(z) f^{\prime \prime}(\varphi(z)) \mid \\
\leq & \left(1-|z|^{2}\right) \log \frac{2}{1-|z|}\left|\psi_{1}^{\prime}(z)\right||f(\varphi(z))| \\
& +\left(1-|z|^{2}\right) \log \frac{2}{1-|z|}\left|\psi_{1}(z) \varphi^{\prime}(z)+\psi_{2}^{\prime}(z)\right|\left|f^{\prime}(\varphi(z))\right| \\
& +\left(1-|z|^{2}\right) \log \frac{2}{1-|z|}\left|\psi_{2}(z) \varphi^{\prime}(z) f^{\prime \prime}(\varphi(z))\right| \\
\leq & C\|f\|_{H^{p}} \frac{\left(1-|z|^{2}\right) \log \frac{2}{1-|z|}\left|\psi_{1}^{\prime}(z)\right|}{\left(1-|\varphi(z)|^{2}\right)^{1 / p}}+C\|f\|_{H^{p}} \frac{\left(1-|z|^{2}\right) \log \frac{2}{1-|z|}\left|\psi_{1}(z) \varphi^{\prime}(z)+\psi_{2}^{\prime}(z)\right|}{\left(1-|\varphi(z)|^{2}\right)^{1+1 / p}} \\
& +C\|f\|_{H^{p}} \frac{\left(1-|z|^{2}\right) \log \frac{2}{1-|z|}\left|\psi_{2}(z) \varphi^{\prime}(z)\right|}{\left(1-|\varphi(z)|^{2}\right)^{2+1 / p}} \\
\leq & C\|f\|_{H^{p}} .
\end{aligned}
$$

On the other hand, by Lemma 2.1 we have

$$
\begin{aligned}
\left|\left(T_{\psi_{1}, \psi_{2}, \varphi} f\right)(0)\right| & =\left|\psi_{1}(0) f(\varphi(0))+\psi_{2}(0) f^{\prime}(\varphi(0))\right| \\
& \leq C\left(\frac{\left|\psi_{1}(0)\right|}{\left(1-|\varphi(0)|^{2}\right)^{1 / p}}+\frac{\left|\psi_{2}(0)\right|}{\left(1-|\varphi(0)|^{2}\right)^{1+1 / p}}\right)\|f\|_{H^{p}} .
\end{aligned}
$$

Applying conditions (3.4) and (3.5), we deduce that the operator $T_{\psi_{1}, \psi_{2}, \varphi}: H^{p} \rightarrow \mathcal{B}_{\log }$ is bounded.

(a) $\Rightarrow$ (b). Now assume that $T_{\psi_{1}, \psi_{2}, \varphi}: H^{p} \rightarrow \mathcal{B}_{\log }$ is bounded. That means that there exists a constant $C$ such that

$$
\left\|T_{\psi_{1}, \psi_{2}, \varphi} f\right\|_{\mathcal{B}_{\log }} \leq C\|f\|_{H^{p}}
$$

for all $f \in H^{p}$. For $f(z)=1 \in H^{p}$, we have

$$
K_{1}:=\sup _{z \in \mathbb{D}}\left(1-|z|^{2}\right) \log \frac{2}{1-|z|}\left|\psi_{1}^{\prime}(z)\right|<\infty .
$$

For $f(z)=z \in H^{p}$, we have

$$
\sup _{z \in \mathbb{D}}\left(1-|z|^{2}\right) \log \frac{2}{1-|z|}\left|\psi_{1}^{\prime}(z) \varphi(z)+\psi_{1}(z) \varphi^{\prime}(z)+\psi_{2}^{\prime}(z)\right|<\infty .
$$


By (3.6), (3.7), the triangle inequality, and the fact that $\|\varphi\|_{\infty} \leq 1$, we obtain

$$
K_{2}:=\sup _{z \in \mathbb{D}}\left(1-|z|^{2}\right) \log \frac{2}{1-|z|}\left|\psi_{1}(z) \varphi^{\prime}(z)+\psi_{2}^{\prime}(z)\right|<\infty .
$$

For $f(z)=z^{2} \in H^{p}$, we get

$$
\begin{aligned}
& \sup _{z \in \mathbb{D}}\left(1-|z|^{2}\right) \log \frac{2}{1-|z|} \mid \psi_{1}^{\prime}(z)(\varphi(z))^{2} \\
& \quad+2\left(\psi_{1}(z) \varphi^{\prime}(z)+\psi_{2}^{\prime}(z)\right) \varphi(z)+2 \psi_{2}(z) \varphi^{\prime}(z) \mid<\infty .
\end{aligned}
$$

From (3.6), (3.8), (3.9), the triangle inequality, and the boundedness of the function $\varphi(z)$, we have

$$
K_{3}:=\sup _{z \in \mathbb{D}}\left(1-|z|^{2}\right) \log \frac{2}{1-|z|}\left|\psi_{2}(z) \varphi^{\prime}(z)\right|<\infty
$$

For a fixed $w \in \mathbb{D}$ and constants $a, b$, we consider the following test functions:

$$
f_{w}(z)=\frac{a\left(1-|w|^{2}\right)}{(1-\bar{w} z)^{1+1 / p}}-\frac{\left(1-|w|^{2}\right)^{2}}{(1-\bar{w} z)^{2+1 / p}}+\frac{b\left(1-|w|^{2}\right)^{3}}{(1-\bar{w} z)^{3+1 / p}}
$$

By the elementary inequality $(s+t)^{p} \leq C\left(s^{p}+t^{p}\right)(s>0, t>0)$ and Lemma 2.2, one has

$$
\begin{aligned}
M_{p}^{p}\left(f_{w}, r\right) & =\frac{1}{2 \pi} \int_{0}^{2 \pi}\left|f_{w}\left(r e^{i \theta}\right)\right|^{p} \mathrm{~d} \theta \\
& \leq C \int_{0}^{2 \pi}\left(\frac{|a|^{p}\left(1-|w|^{2}\right)^{p}}{\left|1-\bar{w} r e^{i \theta}\right|^{1+p}}+\frac{\left(1-|w|^{2}\right)^{2 p}}{\left|1-\bar{w} r e^{i \theta}\right|^{1+2 p}}+\frac{|b|^{p}\left(1-|w|^{2}\right)^{3 p}}{\left|1-\bar{w} r e^{i \theta}\right|^{1+3 p}}\right) \mathrm{d} \theta \\
& \leq C\left(\frac{|a|^{p}\left(1-|w|^{2}\right)^{p}}{(1-|w| r)^{p}}+\frac{\left(1-|w|^{2}\right)^{2 p}}{(1-|w| r)^{2 p}}+\frac{|b|^{p}\left(1-|w|^{2}\right)^{3 p}}{(1-|w| r)^{3 p}}\right),
\end{aligned}
$$

hence $f_{w} \in H^{p}$ and

$$
\sup _{w \in \mathbb{D}}\left\|f_{w}\right\|_{H^{p}} \leq C .
$$

A straightforward calculation shows that

$$
f_{w}^{\prime}(z)=\bar{w}\left(\frac{a A_{1, p}\left(1-|w|^{2}\right)}{(1-\bar{w} z)^{2+1 / p}}-\frac{A_{2, p}\left(1-|w|^{2}\right)^{2}}{(1-\bar{w} z)^{3+1 / p}}+\frac{b A_{3, p}\left(1-|w|^{2}\right)^{3}}{(1-\bar{w} z)^{4+1 / p}}\right)
$$

and

$$
\begin{aligned}
f_{w}^{\prime \prime}(z)= & (\bar{w})^{2}\left(\frac{a A_{1, p} A_{2, p}\left(1-|w|^{2}\right)}{(1-\bar{w} z)^{3+1 / p}}-\frac{A_{2, p} A_{3, p}\left(1-|w|^{2}\right)^{2}}{(1-\bar{w} z)^{4+1 / p}}\right) \\
& +(\bar{w})^{2}\left(\frac{b A_{3, p} A_{4, p}\left(1-|w|^{2}\right)^{3}}{(1-\bar{w} z)^{5+1 / p}}\right),
\end{aligned}
$$

where $A_{j, p}=j+1 / p, j=1,2,3,4$. 
If taking $a=\frac{A_{2, p}}{2 A_{1, p}}, b=\frac{A_{2, p}}{2 A_{3, p}}$ in (3.11), then we have $f_{w}^{\prime}(w)=f_{w}^{\prime \prime}(w)=0$,

$$
f_{w}(w)=\frac{C_{1}(p)}{\left(1-|w|^{2}\right)^{1 / p}}
$$

where $C_{1}(p)=a+b-1 \neq 0$. Thus for $w \in \mathbb{D}$, we have

$$
\begin{aligned}
C \geq & \left\|T_{\psi_{1}, \psi_{2}, \varphi} f_{\varphi(w)}\right\|_{H^{p}} \\
\geq & \sup _{z \in \mathbb{D}}\left(1-|z|^{2}\right) \log \frac{2}{1-|z|}\left|\left(T_{\psi_{1}, \psi_{2}, \varphi} f_{\varphi(w)}\right)^{\prime}(z)\right| \\
= & \sup _{z \in \mathbb{D}}\left(1-|z|^{2}\right) \log \frac{2}{1-|z|} \mid \psi_{1}^{\prime}(z) f_{\varphi(w)}(\varphi(z))+\left(\psi_{1}(z) \varphi^{\prime}(z)+\psi_{2}^{\prime}(z)\right) f_{w}^{\prime}(\varphi(z)) \\
& +\psi_{2}(z) \varphi^{\prime}(z) f_{\varphi(w)}^{\prime \prime}(\varphi(z)) \mid \\
\geq & \left(1-|w|^{2}\right)\left(\log \frac{2}{1-|w|}\right) \mid \psi_{1}^{\prime}(w) f_{\varphi(w)}(\varphi(w))+\left(\psi_{1}(w) \varphi^{\prime}(w)+\psi_{2}^{\prime}(w)\right) f_{\varphi(w)}^{\prime}(\varphi(w)) \\
& +\psi_{2}(w) \varphi^{\prime}(w) f_{\varphi(w)}^{\prime \prime}(\varphi(w)) \mid \\
= & \frac{\left|C_{1}(p)\right|\left(1-|w|^{2}\right)\left(\log \frac{2}{1-|w|}\right)\left|\psi_{1}^{\prime}(w)\right|}{\left(1-|\varphi(w)|^{2}\right)^{1 / p}}
\end{aligned}
$$

from which we get (3.1).

For a fixed $w \in \mathbb{D}$, set

$$
g_{w}(z)=-\frac{\left(1-|w|^{2}\right)}{(1-\bar{w} z)^{1+1 / p}}+\frac{c\left(1-|w|^{2}\right)^{2}}{(1-\bar{w} z)^{2+1 / p}}+\frac{d\left(1-|w|^{2}\right)^{3}}{(1-\bar{w} z)^{3+1 / p}}
$$

It is easy to see that

$$
\begin{aligned}
g_{w}^{\prime}(z)= & \bar{w}\left(\frac{-A_{1, p}\left(1-|w|^{2}\right)}{(1-\bar{w} z)^{2+1 / p}}+\frac{c A_{2, p}\left(1-|w|^{2}\right)^{2}}{(1-\bar{w} z)^{3+1 / p}}+\frac{d A_{3, p}\left(1-|w|^{2}\right)^{3}}{(1-\bar{w} z)^{4+1 / p}}\right), \\
g_{w}^{\prime \prime}(z)= & (\bar{w})^{2}\left(\frac{-A_{1, p} A_{2, p}\left(1-|w|^{2}\right)}{(1-\bar{w} z)^{3+1 / p}}+\frac{c A_{2, p} A_{3, p}\left(1-|w|^{2}\right)^{2}}{(1-\bar{w} z)^{4+1 / p}}\right) \\
& +(\bar{w})^{2}\left(\frac{d A_{3, p} A_{4, p}\left(1-|w|^{2}\right)^{3}}{(1-\bar{w} z)^{5+1 / p}}\right) .
\end{aligned}
$$

By using the same argument in the above, we also have $g_{w} \in H^{p}$ and $\sup _{w \in \mathbb{D}}\left\|g_{w}\right\|_{H^{p}} \leq C$ with a direct calculation. We can take two constants $c$ and $d$ in (3.16) such that $g_{w}(w)=$ $g_{w}^{\prime \prime}(w)=0$, then

$$
g_{w}^{\prime}(w)=\frac{C_{2}(p) \bar{w}}{\left(1-|w|^{2}\right)^{1+1 / p}}
$$

Hence, for $w \in \mathbb{D}$,

$$
\begin{aligned}
C & \geq\left\|T_{\psi_{1}, \psi_{2}, \varphi} g_{\varphi(w)}\right\|_{H^{p}} \\
& \geq \frac{\left(1-|w|^{2}\right)\left(\log \frac{2}{1-|w|}\right)\left|\psi_{1}(w) \varphi^{\prime}(w)+\psi_{2}^{\prime}(w)\right| \mid \overline{\varphi(w) \mid}}{\left(1-|\varphi(w)|^{2}\right)^{1+1 / p}} .
\end{aligned}
$$


Using (3.19) we have

$$
\begin{aligned}
& \sup _{\frac{1}{2}<|\varphi(w)|<1} \frac{\left(1-|w|^{2}\right)\left(\log \frac{2}{1-|w|}\right)\left|\psi_{1}(w) \varphi^{\prime}(w)+\psi_{2}^{\prime}(w)\right|}{\left(1-|\varphi(w)|^{2}\right)^{1+1 / p}} \\
& \leq 2 \sup _{\frac{1}{2}<|\varphi(w)|<1} \frac{\left(1-|w|^{2}\right)\left(\log \frac{2}{1-|w|}\right)\left|\psi_{1}(w) \varphi^{\prime}(w)+\psi_{2}^{\prime}(w)\right| \mid \overline{\varphi(w) \mid}}{\left(1-|\varphi(w)|^{2}\right)^{1+1 / p}} \\
& \leq 2 \sup _{w \in \mathbb{D}} \frac{\left(1-|w|^{2}\right)\left(\log \frac{2}{1-|w|}\right)\left|\psi_{1}(w) \varphi^{\prime}(w)+\psi_{2}^{\prime}(w)\right||\varphi(w)|}{\left(1-|\varphi(w)|^{2}\right)^{1+1 / p}} \\
& \leq 2 C<\infty .
\end{aligned}
$$

According to (3.8), one has

$$
\begin{aligned}
& \sup _{|\varphi(w)| \leq \frac{1}{2}} \frac{\left(1-|w|^{2}\right)\left(\log \frac{2}{1-|w|}\right)\left|\psi_{1}(w) \varphi^{\prime}(w)+\psi_{2}^{\prime}(w)\right|}{\left(1-|\varphi(w)|^{2}\right)^{1+1 / p}} \\
& \leq\left(\frac{4}{3}\right)^{1+1 / p} \sup _{|\varphi(w)| \leq \frac{1}{2}}\left(1-|w|^{2}\right)\left(\log \frac{2}{1-|w|}\right)\left|\psi_{1}(w) \varphi^{\prime}(w)+\psi_{2}^{\prime}(w)\right| \\
& \leq\left(\frac{4}{3}\right)^{1+1 / p} K_{2}<\infty .
\end{aligned}
$$

Thus combining (3.20) and (3.21) we get the condition (3.2).

Next, we prove that (3.3). To see this, for a fixed $w \in \mathbb{D}$, put

$$
h_{w}(z)=\frac{e\left(1-|w|^{2}\right)}{(1-\bar{w} z)^{1+1 / p}}+\frac{f\left(1-|w|^{2}\right)^{2}}{(1-\bar{w} z)^{2+1 / p}}-\frac{\left(1-|w|^{2}\right)^{3}}{(1-\bar{w} z)^{3+1 / p}}
$$

then

$$
h_{w}^{\prime}(z)=\bar{w}\left(\frac{e A_{1, p}\left(1-|w|^{2}\right)}{(1-\bar{w} z)^{2+1 / p}}+\frac{f A_{2, p}\left(1-|w|^{2}\right)^{2}}{(1-\bar{w} z)^{3+1 / p}}-\frac{A_{3, p}\left(1-|w|^{2}\right)^{3}}{(1-\bar{w} z)^{4+1 / p}}\right)
$$

and

$$
\begin{aligned}
h_{w}^{\prime \prime}(z)= & (\bar{w})^{2}\left(\frac{e A_{1, p} A_{2, p}\left(1-|w|^{2}\right)}{(1-\bar{w} z)^{3+1 / p}}+\frac{f A_{2, p} A_{3, p}\left(1-|w|^{2}\right)^{2}}{(1-\bar{w} z)^{4+1 / p}}\right) \\
& -(\bar{w})^{2}\left(\frac{A_{3, p} A_{4, p}\left(1-|w|^{2}\right)^{3}}{(1-\bar{w} z)^{5+1 / p}}\right) .
\end{aligned}
$$

We have $h_{w} \in H^{p}$ and $\sup _{w \in \mathbb{D}}\left\|h_{w}\right\|_{H^{p}} \leq C$. We can take two constants $e$ and $f$ in (3.22) such that $h_{w}(w)=h_{w}^{\prime}(w)=0$, then

$$
h_{w}^{\prime \prime}(w)=\frac{C_{3}(p)(\bar{w})^{2}}{\left(1-|w|^{2}\right)^{2+1 / p}} .
$$

Thus for $w \in \mathbb{D}$, we have

$$
\begin{aligned}
C & \geq\left\|T_{\psi_{1}, \psi_{2}, \varphi} h_{\varphi(w)}\right\|_{\mathcal{B}_{\log }} \\
& \geq \frac{\left(1-|w|^{2}\right)\left(\log \frac{2}{1-|w|}\right)\left|\psi_{2}(w) \varphi^{\prime}(w)\right||\varphi(w)|^{2}}{\left(1-|\varphi(w)|^{2}\right)^{2+1 / p}} .
\end{aligned}
$$


By (3.25), we have

$$
\begin{aligned}
& \sup _{\frac{1}{2}<|\varphi(w)|<1} \frac{\left(1-|w|^{2}\right)\left(\log \frac{2}{1-|w|}\right)\left|\psi_{2}(w) \varphi^{\prime}(w)\right|}{\left(1-|\varphi(w)|^{2}\right)^{2+1 / p}} \\
& \leq \sup _{\frac{1}{2}<|\varphi(w)|<1} \frac{\left(1-|w|^{2}\right)\left(\log \frac{2}{1-|w|}\right)\left|\psi_{2}(w) \varphi^{\prime}(w)\right||\varphi(w)|^{2}}{\left(1-|\varphi(w)|^{2}\right)^{2+1 / p}} \\
& \leq 4 \sup _{w \in \mathbb{D}} \frac{\left(1-|w|^{2}\right)\left(\log \frac{2}{1-|w|}\right)\left|\psi_{2}(w) \varphi^{\prime}(w)\right||\varphi(w)|^{2}}{\left(1-|\varphi(w)|^{2}\right)^{2+1 / p}} \\
& \leq 4 C<\infty, \quad\left(1-|w|^{2}\right)\left(\log \frac{2}{1-|w|}\right)\left|\psi_{2}(w) \varphi^{\prime}(w)\right| \\
& \sup _{|\varphi(w)| \leq \frac{1}{2}} \frac{\left(1-|\varphi(w)|^{2}\right)^{2+1 / p}}{\leq\left(\frac{4}{3}\right)^{2+1 / p} \sup _{|\varphi(w)| \leq \frac{1}{2}}\left(1-|w|^{2}\right)\left(\log \frac{2}{1-|w|}\right)\left|\psi_{2}(w) \varphi^{\prime}(w)\right|} \\
& \leq\left(\frac{4}{3}\right)^{2+1 / p} \sup _{w \in \mathbb{D}}\left(1-|w|^{2}\right)\left(\log \frac{2}{1-|w|}\right)\left|\psi_{2}(w) \varphi^{\prime}(w)\right| \\
& \leq C K_{3}<\infty
\end{aligned}
$$

Combining (3.26) with (3.27) we get (3.3). That ends the proof of Theorem 3.1.

The following corollary follows by setting $\psi_{1}(z)=\psi(z)$ and $\psi_{2}(z)=0$ in Theorem 3.1 at once.

Corollary 3.2 ([10, Theorem 4.1]) Let $\psi \in H(\mathbb{D}), \varphi$ denote an analytic self-map of $\mathbb{D}$, then the weighted composition operator $W_{\psi, \varphi}: H^{p} \rightarrow \mathcal{B}_{\log }$ is a bounded operator if and only if

$$
x_{\psi, \varphi}=\sup _{z \in \mathbb{D}} \frac{\left(1-|z|^{2}\right) \log \frac{2}{1-|z|}\left|\psi^{\prime}(z)\right|}{\left(1-|\varphi(z)|^{2}\right)^{1 / p}}<\infty
$$

and

$$
y_{\psi, \varphi}=\sup _{z \in \mathbb{D}} \frac{\left(1-|z|^{2}\right) \log \frac{2}{1-|z|}\left|\psi(z) \varphi^{\prime}(z)\right|}{\left(1-|\varphi(z)|^{2}\right)^{1+1 / p}}<\infty .
$$

The following corollary follows by setting $\psi_{1}(z)=\psi^{\prime}(z)$ and $\psi_{2}(z)=\psi(z) \varphi(z)$ in Theorem 3.1 at once.

Corollary 3.3 Let $\psi \in H(\mathbb{D}), \varphi$ denote an analytic self-map of $\mathbb{D}$, then the weighted composition followed by differentiation $D W_{\psi, \varphi}: H^{p} \rightarrow \mathcal{B}_{\log }$ is a bounded operator if and only if

$$
\begin{aligned}
& \sup _{z \in \mathbb{D}} \frac{\left(1-|z|^{2}\right) \log \frac{2}{1-|z|}\left|\psi^{\prime \prime}(z)\right|}{\left(1-|\varphi(z)|^{2}\right)^{1 / p}}<\infty, \\
& \sup _{z \in \mathbb{D}} \frac{\left(1-|z|^{2}\right) \log \frac{2}{1-|z|}\left|\psi^{\prime}(z)\left(\varphi^{\prime}(z)+\varphi(z)\right)+\psi(z) \varphi^{\prime}(z)\right|}{\left(1-|\varphi(z)|^{2}\right)^{1+1 / p}}<\infty
\end{aligned}
$$


and

$$
\sup _{z \in \mathbb{D}} \frac{\left(1-|z|^{2}\right) \log \frac{2}{1-|z|}\left|\psi(z) \varphi(z) \varphi^{\prime}(z)\right|}{\left(1-|\varphi(z)|^{2}\right)^{2+1 / p}}<\infty
$$

By using the density of the set of all polynomials in $H^{p}$, we can characterize the boundedness of $T_{\psi_{1}, \psi_{2}, \varphi}: H^{p} \rightarrow \mathcal{B}_{\log , 0}$.

Theorem 3.4 Let $\psi_{1}, \psi_{2} \in H(\mathbb{D}), \varphi$ denote an analytic self-map of $\mathbb{D}$, then $T_{\psi_{1}, \psi_{2}, \varphi}: H^{p} \rightarrow$ $\mathcal{B}_{\log , 0}$ is a bounded operator if and only if $T_{\psi_{1}, \psi_{2}, \varphi}: H^{p} \rightarrow \mathcal{B}_{\log }$ is a bounded operator and

$$
\begin{aligned}
& \lim _{|z| \rightarrow 1}\left(1-|z|^{2}\right) \log \frac{2}{1-|z|}\left|\psi_{1}^{\prime}(z)\right|=0, \\
& \lim _{|z| \rightarrow 1}\left(1-|z|^{2}\right) \log \frac{2}{1-|z|}\left|\psi_{1}(z) \varphi^{\prime}(z)+\psi_{2}^{\prime}(z)\right|=0, \\
& \lim _{|z| \rightarrow 1}\left(1-|z|^{2}\right) \log \frac{2}{1-|z|}\left|\psi_{2}(z) \varphi^{\prime}(z)\right|=0 .
\end{aligned}
$$

Proof For the first half of the theorem we may assume that $T_{\psi_{1}, \psi_{2}, \varphi}: H^{p} \rightarrow \mathcal{B}_{\log , 0}$ is bounded, it is clear that $T_{\psi_{1}, \psi_{2}, \varphi}: H^{p} \rightarrow \mathcal{B}_{\log }$ is bounded. For $f \in H^{p}, T_{\psi_{1}, \psi_{2}, \varphi} f \in \mathcal{B}_{\log , 0}$. Taking $f(z)=1 \in H^{p}$, we have

$$
\lim _{|z| \rightarrow 1}\left(1-|z|^{2}\right) \log \frac{2}{1-|z|}\left|\psi_{1}^{\prime}(z)\right|=0
$$

that is, (3.28) holds. Taking $f(z)=z \in H^{p}$, we get

$$
\lim _{|z| \rightarrow 1}\left(1-|z|^{2}\right) \log \frac{2}{1-|z|}\left|\psi_{1}^{\prime}(z) \varphi(z)+\psi_{1}(z) \varphi^{\prime}(z)+\psi_{2}^{\prime}(z)\right|=0
$$

By (3.31), (3.28), the triangle inequality, and $\|\varphi\|_{\infty} \leq 1$, we have

$$
\lim _{|z| \rightarrow 1}\left(1-|z|^{2}\right) \log \frac{2}{1-|z|}\left|\psi_{1}(z) \varphi^{\prime}(z)+\psi_{2}^{\prime}(z)\right|=0
$$

that is, (3.29) holds. Taking $f(z)=z^{2} \in H^{p}$, one has

$$
\begin{aligned}
& \lim _{|z| \rightarrow 1}\left(1-|z|^{2}\right) \log \frac{2}{1-|z|} \mid \psi_{1}^{\prime}(z)(\varphi(z))^{2} \\
& \quad+2\left(\psi_{1}(z) \varphi^{\prime}(z)+\psi_{2}^{\prime}(z)\right) \varphi(z)+2 \psi_{2}(z) \varphi^{\prime}(z) \mid=0 .
\end{aligned}
$$

Using (3.28), (3.29), (3.32), the triangle inequality, and the boundedness of the function $\varphi(z)$ we have

$$
\lim _{|z| \rightarrow 1}\left(1-|z|^{2}\right) \log \frac{2}{1-|z|}\left|\psi_{2}(z) \varphi^{\prime}(z)\right|=0
$$

Hence (3.30) follows. The proof of the first half of Theorem 3.4 is thus complete. 
As for the proof of the second half, we let $T_{\psi_{1}, \psi_{2}, \varphi}: H^{p} \rightarrow \mathcal{B}_{\log }$ be bounded and (3.28), (3.29), and (3.30) hold. Then for each polynomial $L$, one has

$$
\begin{aligned}
(1- & \left.|z|^{2}\right) \log \frac{2}{1-|z|}\left|\left(T_{\psi_{1}, \psi_{2}, \varphi} L\right)^{\prime}(z)\right| \\
= & \left(1-|z|^{2}\right) \log \frac{2}{1-|z|} \mid \psi_{1}^{\prime}(z) L(\varphi(z))+\left(\psi_{1}(z) \varphi^{\prime}(z)+\psi_{2}^{\prime}(z)\right) L^{\prime}(\varphi(z)) \\
& +\psi_{2}(z) \varphi^{\prime}(z) L^{\prime \prime}(\varphi(z)) \mid \\
\leq & \left(1-|z|^{2}\right) \log \frac{2}{1-|z|}\left|\psi_{1}^{\prime}(z)\right||L(\varphi(z))| \\
& +\left(1-|z|^{2}\right) \log \frac{2}{1-|z|}\left|\psi_{1}(z) \varphi^{\prime}(z)+\psi_{2}^{\prime}(z)\right|\left|L^{\prime}(\varphi(z))\right| \\
& +\left(1-|z|^{2}\right) \log \frac{2}{1-|z|}\left|\psi_{2}(z) \varphi^{\prime}(z)\right|\left|L^{\prime \prime}(\varphi(z))\right| \\
\leq & \left(1-|z|^{2}\right) \log \frac{2}{1-|z|}\left|\psi_{1}^{\prime}(z)\right|\|L\|_{\infty} \\
& +\left(1-|z|^{2}\right) \log \frac{2}{1-|z|}\left|\psi_{1}(z) \varphi^{\prime}(z)+\psi_{2}^{\prime}(z)\right|\left\|L^{\prime}\right\|_{\infty} \\
& +\left(1-|z|^{2}\right) \log \frac{2}{1-|z|}\left|\psi_{2}(z) \varphi^{\prime}(z)\right|\left\|L^{\prime \prime}\right\|_{\infty} \\
\rightarrow & 0 \quad \text { as }|z| \rightarrow 1,
\end{aligned}
$$

from which it follows that $T_{\psi_{1}, \psi_{2}, \varphi} L \in \mathcal{B}_{\log , 0}$. Since the set of all polynomials is dense in $H^{p}$, thus for each $f \in H^{p}$, there is a sequence of polynomials $\left\{L_{k}\right\}_{k \in \mathbb{N}}$, such that

$$
\lim _{k \rightarrow \infty}\left\|L_{k}-f\right\|_{H^{p}}=0
$$

so

$$
\left\|T_{\psi_{1}, \psi_{2}, \varphi} L_{k}-T_{\psi_{1}, \psi_{2}, \varphi} f\right\|_{\mathcal{B}_{\log }} \leq\left\|T_{\psi_{1}, \psi_{2}, \varphi}\right\|\left\|L_{k}-f\right\|_{H^{p}} \rightarrow 0 \quad \text { as } k \rightarrow \infty
$$

Since $\mathcal{B}_{\log , 0}$ is the closed subset of $\mathcal{B}_{\log }$, we see that $T_{\psi_{1}, \psi_{2}, \varphi} f \in \mathcal{B}_{\log , 0}$, and consequently $T_{\psi_{1}, \psi_{2}, \varphi}\left(H^{p}\right) \subseteq \mathcal{B}_{\log , 0}$, the boundedness of the operator $T_{\psi_{1}, \psi_{2}, \varphi}: H^{p} \rightarrow \mathcal{B}_{\log }$ implies that $T_{\psi_{1}, \psi_{2}, \varphi}: H^{p} \rightarrow \mathcal{B}_{\log , 0}$ is bounded. This ends the proof of Theorem 3.4.

According to Theorem 3.4 we immediately get the following.

Corollary 3.5 ([10, Theorem 5.1$])$ Let $\psi \in H(\mathbb{D}), \varphi$ denotes an analytic self-map of $\mathbb{D}$, then the weighted composition operator $W_{\psi, \varphi}: H^{p} \rightarrow \mathcal{B}_{\log , 0}$ is bounded if and only if $W_{\psi, \varphi}$ : $H^{p} \rightarrow \mathcal{B}_{\log }$ is bounded, $\psi \in \mathcal{B}_{\log , 0}$, and

$$
\lim _{|z| \rightarrow 1}\left(1-|z|^{2}\right) \log \frac{2}{1-|z|}\left|\psi(z) \varphi^{\prime}(z)\right|=0 .
$$

Corollary 3.6 Let $\psi \in H(\mathbb{D})$, $\varphi$ denotes an analytic self-map of $\mathbb{D}$, then the weighted composition followed by differentiation $D W_{\psi, \varphi}: H^{p} \rightarrow \mathcal{B}_{\log , 0}$ is a bounded operator if and only 


$$
\begin{aligned}
& \text { if } D W_{\psi, \varphi}: H^{p} \rightarrow \mathcal{B}_{\log } \text { is bounded and } \\
& \lim _{|z| \rightarrow 1}\left(1-|z|^{2}\right) \log \frac{2}{1-|z|}\left|\psi^{\prime \prime}(z)\right|=0, \\
& \lim _{|z| \rightarrow 1}\left(1-|z|^{2}\right) \log \frac{2}{1-|z|}\left|\psi^{\prime}(z)\left(\varphi^{\prime}(z)+\varphi(z)\right)+\psi(z) \varphi^{\prime}(z)\right|=0, \\
& \lim _{|z| \rightarrow 1}\left(1-|z|^{2}\right) \log \frac{2}{1-|z|}\left|\psi(z) \varphi(z) \varphi^{\prime}(z)\right|=0 .
\end{aligned}
$$

4 The compactness of the operator $T_{\psi_{1}, \psi_{2}, \varphi}: H^{p} \rightarrow \mathcal{B}_{\log }\left(\mathcal{B}_{\log , 0}\right)$

Now we are in a position to prove compactness.

Theorem 4.1 Let $\psi_{1}, \psi_{2} \in H(\mathbb{D}), \varphi$ denote an analytic self-map of $\mathbb{D}$, then the following statements are equivalent.

(a) $T_{\psi_{1}, \psi_{2}, \varphi}: H^{p} \rightarrow \mathcal{B}_{\log }$ is compact;

(b) $T_{\psi_{1}, \psi_{2}, \varphi}: H^{p} \rightarrow \mathcal{B}_{\log }$ is bounded and

$$
\begin{aligned}
& \lim _{|\varphi(z)| \rightarrow 1} \frac{\left(1-|z|^{2}\right) \log \frac{2}{1-|z|}\left|\psi_{1}^{\prime}(z)\right|}{\left(1-|\varphi(z)|^{2}\right)^{1 / p}}=0, \\
& \lim _{|\varphi(z)| \rightarrow 1} \frac{\left(1-|z|^{2}\right) \log \frac{2}{1-|z|}\left|\psi_{1}(z) \varphi^{\prime}(z)+\psi_{2}^{\prime}(z)\right|}{\left(1-|\varphi(z)|^{2}\right)^{1+1 / p}}=0, \\
& \lim _{|\varphi(z)| \rightarrow 1} \frac{\left(1-|z|^{2}\right) \log \frac{2}{1-|z|}\left|\psi_{2}(z) \varphi^{\prime}(z)\right|}{\left(1-|\varphi(z)|^{2}\right)^{2+1 / p}}=0 .
\end{aligned}
$$

Proof (b) $\Rightarrow$ (a). Suppose that $T_{\psi_{1}, \psi_{2}, \varphi}: H^{p} \rightarrow \mathcal{B}_{\log }$ is bounded, (4.1), (4.2), and (4.3) hold. To prove that $T_{\psi_{1}, \psi_{2}, \varphi}: H^{p} \rightarrow \mathcal{B}_{\log }$ is compact, for any bounded sequence $\left\{f_{k}\right\}$ in $H^{p}$ with $f_{k} \rightarrow 0$ uniformly on compact subsets of $\mathbb{D}$, let $\left\|f_{k}\right\|_{H^{p}} \leq 1$, it suffices, in view of Lemma 2.3, to show that

$$
\left\|T_{\psi_{1}, \psi_{2}, \varphi} f_{k}\right\|_{\mathcal{B}_{\log }} \rightarrow 0 \quad \text { as } k \rightarrow \infty
$$

By (4.1), (4.2), and (4.3), we have for any $\varepsilon>0$, there exists $\rho \in(0,1)$ such that

$$
\begin{aligned}
& \frac{\left(1-|z|^{2}\right) \log \frac{2}{1-|z|}\left|\psi_{1}^{\prime}(z)\right|}{\left(1-|\varphi(z)|^{2}\right)^{1 / p}}<\varepsilon \\
& \frac{\left(1-|z|^{2}\right) \log \frac{2}{1-|z|}\left|\psi_{1}(z) \varphi^{\prime}(z)+\psi_{2}^{\prime}(z)\right|}{\left(1-|\varphi(z)|^{2}\right)^{1+1 / p}}<\varepsilon
\end{aligned}
$$

and

$$
\frac{\left(1-|z|^{2}\right) \log \frac{2}{1-|z|}\left|\psi_{2}(z) \varphi^{\prime}(z)\right|}{\left(1-|\varphi(z)|^{2}\right)^{2+1 / p}}<\varepsilon,
$$

for $\rho<|\varphi(z)|<1$. From the boundedness of the operator $T_{\psi_{1}, \psi_{2}, \varphi}: H^{p} \rightarrow \mathcal{B}_{\log }$ and the proof of Theorem 3.1, (3.6), (3.8), and (3.10) hold. Since $f_{k} \rightarrow 0$ uniformly on compact subsets of $\mathbb{D}$, Cauchy's estimate shows that $f_{k}^{\prime}$ and $f_{k}^{\prime \prime}$ converge to 0 uniformly on compact subsets 
of $\mathbb{D}$, there exists a $K_{0} \in \mathbb{N}$ such that $k>K_{0}$ implies that

$$
\begin{aligned}
& \left|\left(T_{\psi_{1}, \psi_{2}, \varphi} f_{k}\right)(0)\right|+\sup _{|\varphi(z)| \leq \rho}\left(1-|z|^{2}\right) \log \frac{2}{1-|z|}\left|\left(T_{\psi_{1}, \psi_{2}, \varphi} f_{k}\right)^{\prime}(z)\right| \\
& \leq\left|\psi_{1}(0) f_{k}(\varphi(0))+\psi_{2}(0) f_{k}^{\prime}(\varphi(0))\right| \\
& \quad+\sup _{|\varphi(z)| \leq \rho}\left(1-|z|^{2}\right) \log \frac{2}{1-|z|}\left|\psi_{1}^{\prime}(z)\right|\left|f_{k}(\varphi(z))\right| \\
& \quad+\sup _{|\varphi(z)| \leq \rho}\left(1-|z|^{2}\right) \log \frac{2}{1-|z|}\left|\psi_{1}(z) \varphi^{\prime}(z)+\psi_{2}^{\prime}(z)\right|\left|f_{k}^{\prime}(\varphi(z))\right| \\
& \quad+\sup _{|\varphi(z)| \leq \rho}\left(1-|z|^{2}\right) \log \frac{2}{1-|z|}\left|\psi_{2}(z) \varphi^{\prime}(z)\right|\left|f_{k}^{\prime \prime}(\varphi(z))\right| \\
& \leq\left|\psi_{1}(0)\right|\left|f_{k}(\varphi(0))\right|+\left|\psi_{2}(0) f_{k}^{\prime}(\varphi(0))\right| \\
& \quad+K_{1} \sup _{|\varphi(z)| \leq \rho}\left|f_{k}(\varphi(z))\right|+K_{2} \sup _{|\varphi(z)| \leq \rho}\left|f_{k}^{\prime}(\varphi(z))\right|+K_{3} \sup _{|\varphi(z)| \leq \rho}\left|f_{k}^{\prime \prime}(\varphi(z))\right| \\
& <C \varepsilon .
\end{aligned}
$$

When $k>K_{0}$, from (4.4), (4.5), (4.6), (4.7), Lemmas 2.1 and 2.2, one has

$$
\begin{aligned}
\| & T_{\psi_{1}, \psi_{2}, \varphi} f_{k} \|_{\mathcal{B}_{\log }} \\
= & \left|\left(T_{\psi_{1}, \psi_{2}, \varphi} f_{k}\right)(0)\right|+\sup _{z \in \mathbb{D}}\left(1-|z|^{2}\right) \log \frac{2}{1-|z|}\left|\left(T_{\psi_{1}, \psi_{2}, \varphi} f_{k}\right)^{\prime}(z)\right| \\
\leq & \left(\left|\left(T_{\psi_{1}, \psi_{2}, \varphi} f_{k}\right)(0)\right|+\sup _{|\varphi(z)| \leq \rho}\left(1-|z|^{2}\right) \log \frac{2}{1-|z|}\left|\left(T_{\psi_{1}, \psi_{2}, \varphi} f_{k}\right)^{\prime}(z)\right|\right) \\
& +\sup _{\rho<|\varphi(z)|<1}\left(1-|z|^{2}\right) \log \frac{2}{1-|z|}\left|\left(T_{\psi_{1}, \psi_{2}, \varphi} f_{k}\right)^{\prime}(z)\right| \\
< & C \varepsilon+2 C \sup _{\rho<|\varphi(z)|<1} \frac{\left(1-|z|^{2}\right) \log \frac{2}{1-|z|}\left|\psi_{1}^{\prime}(z)\right|}{\left(1-|\varphi(z)|^{2}\right)^{1 / p}} \mid f_{k} \|_{H^{p}} \\
& +C \sup _{\rho<|\varphi(z)|<1} \frac{\left(1-|z|^{2}\right) \log \frac{2}{1-|z|}\left|\psi_{1}(z) \varphi^{\prime}(z)+\psi_{2}^{\prime}(z)\right|}{\left(1-|\varphi(z)|^{2}\right)^{1+1 / p}}\left\|f_{k}\right\|_{H^{p}} \\
& +C \sup _{\rho<|\varphi(z)|<1} \frac{\left(1-|z|^{2}\right) \log \frac{2}{1-|z|}\left|\psi_{2}(z) \varphi^{\prime}(z)\right|}{\left(1-|\varphi(z)|^{2}\right)^{2+1 / p}}\left\|f_{k}\right\|_{H^{p}} \\
< & 5 C \varepsilon, \quad
\end{aligned}
$$

it follows that the operator $T_{\psi_{1}, \psi_{2}, \varphi}: \mathcal{B}_{\log } \rightarrow \mathcal{B}_{\log }$ is compact.

(a) $\Rightarrow$ (b). It is clear that the compactness of $T_{\psi_{1}, \psi_{2}, \varphi}: H^{p} \rightarrow \mathcal{B}_{\log }$ implies the boundedness of $T_{\psi_{1}, \psi_{2}, \varphi}: H^{p} \rightarrow \mathcal{B}_{\log }$. If $\|\varphi\|_{\infty}<1$, it is clear that the limit in (4.1), (4.2), and (4.3) is vacuously equal to zero. Hence, assume that $\|\varphi\|_{\infty}=1$, let $\left\{z_{k}\right\}$ be a sequence in $\mathbb{D}$ such that $\left|\varphi\left(z_{k}\right)\right| \rightarrow 1$ as $k \rightarrow \infty$. We can use the test functions

$$
f_{k}(z)=f_{\varphi\left(z_{k}\right)}(z)
$$

$f_{w}$ here is defined in (3.11). Equations (3.12), (3.13), and (3.14) tell us that $\sup _{k \in \mathbb{N}}\left\|f_{k}\right\|_{H^{p}} \leq C$ 
and

$$
f_{k}\left(\varphi\left(z_{k}\right)\right)=\frac{C_{1}(p)}{\left(1-\left|\varphi\left(z_{k}\right)\right|^{2}\right)^{1 / p}}, \quad f_{k}^{\prime}\left(\varphi\left(z_{k}\right)\right)=f_{k}^{\prime \prime}\left(\varphi\left(z_{k}\right)\right)=0 .
$$

We see that $f_{k}$ converges to 0 uniformly on $\mathbb{D}$, hence $f_{k}$ converges to 0 uniformly on compact subsets of $\mathbb{D}$. Then $f_{k}$ is a bounded sequence in $H^{p}$ which converges to 0 uniformly on compact subsets of $\mathbb{D}$. By Lemma 2.3 , we obtain

$$
\lim _{k \rightarrow \infty}\left\|T_{\psi_{1}, \psi_{2}, \varphi} f_{k}\right\|_{\mathcal{B}_{\log }}=0
$$

From (3.15) and the compactness of $T_{\psi_{1}, \psi_{2}, \varphi}: H^{p} \rightarrow \mathcal{B}_{\text {log }}$, we get

$$
\begin{aligned}
& \frac{\left|C_{1}(p)\right|\left(1-\left|z_{k}\right|^{2}\right)\left(\log \frac{2}{1-\left|z_{k}\right|}\right)\left|\psi_{1}^{\prime}\left(z_{k}\right)\right|}{\left(1-\left|\varphi\left(z_{k}\right)\right|^{2}\right)^{1 / p}} \\
& \leq\left\|T_{\psi_{1}, \psi_{2}, \varphi} f_{k}\right\|_{\mathcal{B}_{\log }} \rightarrow 0 \quad \text { as } k \rightarrow \infty .
\end{aligned}
$$

This proves (4.1).

Next, let

$$
g_{k}(z)=g_{\varphi\left(z_{k}\right)}(z)
$$

where $g_{w}$ is defined in (3.16). By a direct calculation, we find that $g_{k}$ converges to 0 uniformly on compact subsets of $\mathbb{D}, g_{k} \in H^{p}$, and $\sup _{k \in \mathbb{N}}\left\|g_{k}\right\|_{H^{p}} \leq C$. By Lemma 2.3, we have

$$
\lim _{k \rightarrow \infty}\left\|T_{\psi_{1}, \psi_{2}, \varphi} g_{k}\right\|_{\mathcal{B}_{\log }}=0
$$

Note that from (3.16), (3.17), and (3.18), one has $g_{k}\left(\varphi\left(z_{k}\right)\right)=g_{k}^{\prime}\left(\varphi\left(z_{k}\right)\right)=0$,

$$
g_{k}^{\prime}\left(\varphi\left(z_{k}\right)\right)=\frac{C_{2}(p) \overline{\varphi\left(z_{k}\right)}}{\left(1-\left|\varphi\left(z_{k}\right)\right|^{2}\right)^{1+1 / p}}
$$

From (3.19) and using the compactness of the operator $T_{\psi_{1}, \psi_{2}, \varphi}: H^{p} \rightarrow \mathcal{B}_{\text {log }}$, we get that

$$
\begin{aligned}
& \frac{\left|C_{2}(p)\right|\left(1-\left|z_{k}\right|^{2}\right)\left(\log \frac{2}{1-\left|z_{k}\right|}\right)\left|\psi_{1}\left(z_{k}\right) \varphi^{\prime}\left(z_{k}\right)+\psi_{2}^{\prime}\left(z_{k}\right)\right|\left|\overline{\varphi\left(z_{k}\right)}\right|}{\left(1-\left|\varphi\left(z_{k}\right)\right|^{2}\right)^{1+1 / p}} \\
& \leq\left\|T_{\psi_{1}, \psi_{2}, \varphi} g_{k}\right\|_{\mathcal{B}_{\log }} \rightarrow 0 \quad \text { as } k \rightarrow \infty .
\end{aligned}
$$

By (4.8) and $\left|\varphi\left(z_{k}\right)\right| \rightarrow 1$, we have

$$
\lim _{k \rightarrow \infty} \frac{\left(1-\left|z_{k}\right|^{2}\right)\left(\log \frac{2}{1-\left|z_{k}\right|}\right)\left|\psi_{1}\left(z_{k}\right) \varphi^{\prime}\left(z_{k}\right)+\psi_{2}^{\prime}\left(z_{k}\right)\right|}{\left(1-\left|\varphi\left(z_{k}\right)\right|^{2}\right)^{1+1 / p}}=0,
$$

it implies that (4.2) holds.

Analogously, (4.3) can be proved by choosing the test function $h_{k}(z)=h_{\varphi\left(z_{k}\right)}(z), h_{w}$ here is defined in (3.22), and that proves Theorem 4.1. 
From Theorem 4.1 we can get the characterization of the compactness of the weighted composition operator $W_{\psi, \varphi}: H^{p} \rightarrow \mathcal{B}_{\log }$ and the operator $D W_{\psi, \varphi}: H^{p} \rightarrow \mathcal{B}_{\log }$.

Corollary 4.2 ([10, Theorem 4.2]) Let $\psi \in H(\mathbb{D})$, $\varphi$ denote an analytic self-map of $\mathbb{D}$, then the weighted composition operator $W_{\psi, \varphi}: H^{p} \rightarrow \mathcal{B}_{\log }$ is compact if and only if $W_{\psi, \varphi}: H^{p} \rightarrow$ $\mathcal{B}_{\log }$ is bounded and

$$
\begin{aligned}
& \lim _{|\varphi(z)| \rightarrow 1} \frac{\left(1-|z|^{2}\right) \log \frac{2}{1-|z|}\left|\psi^{\prime}(z)\right|}{\left(1-|\varphi(z)|^{2}\right)^{1 / p}}=0, \\
& \lim _{|\varphi(z)| \rightarrow 1} \frac{\left(1-|z|^{2}\right) \log \frac{2}{1-|z|}\left|\psi(z) \varphi^{\prime}(z)\right|}{\left(1-|\varphi(z)|^{2}\right)^{1+1 / p}}=0 .
\end{aligned}
$$

Corollary 4.3 Let $\psi \in H(\mathbb{D})$, $\varphi$ denote an analytic self-map of $\mathbb{D}$, then the weighted composition followed by differentiation $D W_{\psi, \varphi}: H^{p} \rightarrow \mathcal{B}_{\log }$ is a compact operator if and only if $D W_{\psi, \varphi}: H^{p} \rightarrow \mathcal{B}_{\log }$ is bounded and

$$
\begin{aligned}
& \lim _{|\varphi(z)| \rightarrow 1} \frac{\left(1-|z|^{2}\right) \log \frac{2}{1-|z|}\left|\psi^{\prime \prime}(z)\right|}{\left(1-|\varphi(z)|^{2}\right)^{1 / p}}=0, \\
& \lim _{|\varphi(z)| \rightarrow 1} \frac{\left(1-|z|^{2}\right) \log \frac{2}{1-|z|}\left|\psi^{\prime}(z)\left(\varphi^{\prime}(z)+\varphi(z)\right)+\psi(z) \varphi^{\prime}(z)\right|}{\left(1-|\varphi(z)|^{2}\right)^{1+1 / p}}=0, \\
& \lim _{|\varphi(z)| \rightarrow 1} \frac{\left(1-|z|^{2}\right) \log \frac{2}{1-|z|}\left|\psi(z) \varphi^{\prime}(z)\right|}{\left(1-|\varphi(z)|^{2}\right)^{2+1 / p}}=0 .
\end{aligned}
$$

Next we consider the compactness of $T_{\psi_{1}, \psi_{2}, \varphi}: H^{p} \rightarrow \mathcal{B}_{\log , 0}$. The compactness of operators of which the range is in $\mathcal{B}_{\log , 0}$ has a close relation with Lemma 2.4 .

Theorem 4.4 Let $\psi_{1}, \psi_{2} \in H(\mathbb{D}), \varphi$ denote an analytic self-map of $\mathbb{D}$, then the following statements are equivalent.

(a) $T_{\psi_{1}, \psi_{2}, \varphi}: H^{p} \rightarrow \mathcal{B}_{\log , 0}$ is compact;

(b)

$$
\begin{aligned}
& \lim _{|z| \rightarrow 1} \frac{\left(1-|z|^{2}\right) \log \frac{2}{1-|z|}\left|\psi_{1}^{\prime}(z)\right|}{\left(1-|\varphi(z)|^{2}\right)^{1 / p}}=0, \\
& \lim _{|z| \rightarrow 1} \frac{\left(1-|z|^{2}\right) \log \frac{2}{1-|z|}\left|\psi_{1}(z) \varphi^{\prime}(z)+\psi_{2}^{\prime}(z)\right|}{\left(1-|\varphi(z)|^{2}\right)^{1+1 / p}}=0
\end{aligned}
$$

and

$$
\lim _{|z| \rightarrow 1} \frac{\left(1-|z|^{2}\right) \log \frac{2}{1-|z|}\left|\psi_{2}(z) \varphi^{\prime}(z)\right|}{\left(1-|\varphi(z)|^{2}\right)^{2+1 / p}}=0 .
$$

Proof (b) $\Rightarrow$ (a). Suppose that (4.9), (4.10), and (4.11) hold. By Theorems 3.1 and 3.4, it is clear that $T_{\psi_{1}, \psi_{2}, \varphi}: H^{p} \rightarrow \mathcal{B}_{\log , 0}$ is bounded. Taking the supremum in inequality (3.4) over all $f \in H^{p}$ such that $\|f\|_{H^{p}} \leq 1$ and letting $|z| \rightarrow 1$, yields

$$
\lim _{|z| \rightarrow 1} \sup _{\|f\|_{H^{p}} \leq 1}\left(1-|z|^{2}\right) \log \frac{2}{1-|z|}\left|\left(T_{\psi_{1}, \psi_{2}, \varphi} f\right)^{\prime}(z)\right|=0 .
$$

Therefore, by Lemma 2.4, we have $T_{\psi_{1}, \psi_{2}, \varphi}: H^{p} \rightarrow \mathcal{B}_{\log , 0}$ is compact. 
(a) $\Rightarrow$ (b). Assume that $T_{\psi_{1}, \psi_{2}, \varphi}: H^{p} \rightarrow \mathcal{B}_{\log , 0}$ is compact. Firstly, it is obvious $T_{\psi_{1}, \psi_{2}, \varphi}$ : $H^{p} \rightarrow \mathcal{B}_{\log }$ is compact. By Theorem 4.1, $\psi_{1}, \psi_{2}$, and $\varphi$ satisfy conditions (4.1), (4.2), and (4.3). It follows that for every $\varepsilon>0$, there exists $\rho \in(0,1)$ such that (4.4), (4.5), and (4.6) hold for $\rho<|\varphi(z)|<1$. On the other hand, since $T_{\psi_{1}, \psi_{2}, \varphi}: H^{p} \rightarrow \mathcal{B}_{\log , 0}$ is compact, then $T_{\psi_{1}, \psi_{2}, \varphi}: H^{p} \rightarrow \mathcal{B}_{\log , 0}$ is bounded. By Theorem 3.4, $\psi_{1}, \psi_{2}$, and $\varphi$ also satisfy conditions (3.28), (3.29), and (3.30). Thus for $\varepsilon>0$, there exists $\gamma \in(0,1)$ such that

$$
\begin{aligned}
& \left(1-|z|^{2}\right) \log \frac{2}{1-|z|}\left|\psi_{1}^{\prime}(z)\right|<\left(1-\rho^{2}\right)^{1 / p} \varepsilon \\
& \left(1-|z|^{2}\right) \log \frac{2}{1-|z|}\left|\psi_{1}(z) \varphi^{\prime}(z)+\psi_{2}^{\prime}(z)\right|<\left(1-\rho^{2}\right)^{1+1 / p} \varepsilon, \\
& \left(1-|z|^{2}\right) \log \frac{2}{1-|z|}\left|\psi_{2}(z) \varphi^{\prime}(z)\right|<\left(1-\rho^{2}\right)^{2+1 / p} \varepsilon
\end{aligned}
$$

for $\gamma<|z|<1$. Next, we prove that (4.12) and (4.4) imply (4.9). The proof of (4.10) and (4.11) is similar, hence it will be omitted.

From (4.4), one has, when $\gamma<|z|<1$ and $\rho<|\varphi(z)|<1$,

$$
\frac{\left(1-|z|^{2}\right) \log \frac{2}{1-|z|}\left|\psi^{\prime}(z)\right|}{\left(1-|\varphi(z)|^{2}\right)^{1 / p}}<\varepsilon .
$$

By (4.12), we get, when $\gamma<|z|<1$ and $|\varphi(z)| \leq \rho$,

$$
\begin{aligned}
& \frac{\left(1-|z|^{2}\right) \log \frac{2}{1-|z|}\left|\psi^{\prime}(z)\right|}{\left(1-|\varphi(z)|^{2}\right)^{1 / p}} \\
& \leq \frac{\left(1-|z|^{2}\right) \log \frac{2}{1-|z|}\left|\psi^{\prime}(z)\right|}{\left(1-\rho^{2}\right)^{1 / p}}<\varepsilon .
\end{aligned}
$$

Having in mind (4.15) and (4.16) we conclude that (4.9) holds. This finishes the proof.

Due to Theorem 4.4, the characterization of the compactness of the weighted composition operator $W_{\psi, \varphi}: H^{p} \rightarrow \mathcal{B}_{\log , 0}$ and the operator $D W_{\psi, \varphi}: H^{p} \rightarrow \mathcal{B}_{\log , 0}$ are now obvious.

Corollary 4.5 ([10, Theorem 5.2]) Let $\psi \in H(\mathbb{D}), \varphi$ denote an analytic self-map of $\mathbb{D}$, then the weighted composition operator $W_{\psi, \varphi}: H^{p} \rightarrow \mathcal{B}_{\log , 0}$ is compact if and only if

$$
\lim _{z \mid \rightarrow 1} \frac{\left(1-|z|^{2}\right) \log \frac{2}{1-|z|}\left|\psi^{\prime}(z)\right|}{\left(1-|\varphi(z)|^{2}\right)^{1 / p}}=0
$$

and

$$
\lim _{|z| \rightarrow 1} \frac{\left(1-|z|^{2}\right) \log \frac{2}{1-|z|}\left|\psi(z) \varphi^{\prime}(z)\right|}{\left(1-|\varphi(z)|^{2}\right)^{1+1 / p}}=0 .
$$

Corollary 4.6 Let $\psi \in H(\mathbb{D}), \varphi$ denote an analytic self-map of $\mathbb{D}$, then the weighted composition followed by differentiation $D W_{\psi, \varphi}: H^{p} \rightarrow \mathcal{B}_{\log , 0}$ is a compact operator if and only 
if

$$
\begin{aligned}
& \lim _{|z| \rightarrow 1} \frac{\left(1-|z|^{2}\right) \log \frac{2}{1-|z|}\left|\psi^{\prime \prime}(z)\right|}{\left(1-|\varphi(z)|^{2}\right)^{1 / p}}=0, \\
& \lim _{|z| \rightarrow 1} \frac{\left(1-|z|^{2}\right) \log \frac{2}{1-|z|}\left|\psi^{\prime}(z)\left(\varphi^{\prime}(z)+\varphi(z)\right)+\psi(z) \varphi^{\prime}(z)\right|}{\left(1-|\varphi(z)|^{2}\right)^{1+1 / p}}=0,
\end{aligned}
$$

and

$$
\lim _{|z| \rightarrow 1} \frac{\left(1-|z|^{2}\right) \log \frac{2}{1-|z|}\left|\psi(z) \varphi(z) \varphi^{\prime}(z)\right|}{\left(1-|\varphi(z)|^{2}\right)^{2+1 / p}}=0
$$

\section{Competing interests}

The authors declare that they have no competing interests.

\section{Authors' contributions}

All authors contributed equally to the writing of this paper. They also read and approved the final manuscript.

\section{Author details}

${ }^{1}$ School of Mathematics and Statistics, Jiangsu Normal University, Xuzhou, 221116, China. ${ }^{2}$ School of Mathematics and Physics Science, Xuzhou Institute of Technology, Xuzhou, 221000, China.

\section{Acknowledgements}

The project is supported by the Natural Science Foundation of China (No. 11171285) and the Priority Academic Program Development of Jiangsu Higher Education Institutions. The authors thank the referees for their valuable suggestions and for bringing important references to our attention.

Received: 25 November 2014 Accepted: 31 December 2014 Published online: 22 January 2015

\section{References}

1. Duren, P: Theory of $H^{P}$ Spaces. Academic Press, New York (1970)

2. Garnett, J: Bounded Analytic Functions, (revised first edition). Graduate Texts in Mathematics, vol. 236. Springer, New York (2007)

3. Ye, S: Multipliers and cyclic vectors on the weighted Bloch type space. Math. J. Okayama Univ. 48, 135-143 (2006)

4. Yoneda, R: The composition operators on weighted Bloch space. Arch. Math. (Basel) 78(4), 310-317 (2002)

5. Rudin, W: Real and Complex Analysis, 2nd edn. McGraw-Hill, New York (1974)

6. Arazy, J: Multipliers of Bloch Functions. University of Haifa Mathematics Publications Series, vol. 54 (1982)

7. Brown, L, Shields, AL: Multipliers and cyclic vectors in the Bloch space. Mich. Math. J. 38, 141-146 (1991)

8. Li, S, Stević, S: Volterra-type operators on Zygmund spaces. J. Inequal. Appl. 2007, Article ID 32124 (2007)

9. Attele, K: Toeplitz and Hankel operators on Bergman one space. Hokkaido Math. J. 21(2), 279-293 (1992)

10. Colonna, F, Li, S: Weighted composition operators from Hardy spaces into logarithmic Bloch spaces. J. Funct. Spaces Appl. 2012, Article ID 454820 (2012)

11. Castillo, RE, Clahane, DD, Farías López, JF, Ramos Fernández, JC: Composition operators from logarithmic Bloch spaces to weighted Bloch spaces. Appl. Math. Comput. 219(12), 6692-6706 (2013)

12. Galanopoulos, P: On $\mathcal{B}_{\log }$ to $Q_{\log }^{p}$ pullbacks. J. Math. Anal. Appl. 337(1), 712-725 (2008)

13. García Ortiz, AJ, Ramos-Fernández, JC: Composition operators from logarithmic Bloch spaces to Bloch-type spaces. Georgian Math. J. 20(4), 671-686 (2013)

14. Petrov, A: Reverse estimates in logarithmic Bloch spaces. Arch. Math. (Basel) 100(6), 551-560 (2013)

15. Qu, H, Liu, Y, Cheng, S: Weighted differentiation composition operator from logarithmic Bloch spaces to Zygmund-type spaces. Abstr. Appl. Anal. 2014, Article ID 832713 (2014)

16. Ye, S: Weighted composition operator between different weighted Bloch type spaces. Acta Math. Sin., Chin. Ser. 50 927-942 (2007)

17. Ye, S: Weighted composition operator from the general function space $F(p, q, s)$ into the logarithmic Bloch space. J. Korean Math. Soc. 45(4), 977-991 (2008)

18. Ye, S: A weighted composition operator on the logarithmic Bloch space. Bull. Korean Math. Soc. 47(3), 527-540 (2010)

19. Stević, S, Sharma, A, Bhat, A: Products of multiplication, composition and differentiation operators on weighted Bergman space. Appl. Math. Comput. 217, 8115-8125 (2011)

20. Stević, S, Sharma, A, Bhat, A: Essential norm of products of multiplication composition and differentiation operators on weighted Bergman spaces. Appl. Math. Comput. 218(6), 2386-2397 (2011)

21. Zhang, F, Liu, Y: Products of multiplication, composition and differentiation operators from mixed-norm spaces to weighted-type spaces. Taiwan. J. Math. 18(6), 1927-1940 (2014)

22. Datt, G: Product of composition and multiplication operators. Acta Math. Vietnam. 37(2), 293-300 (2012)

23. Li, S, Stević, S: Composition followed by differentiation between Bloch type spaces. J. Comput. Anal. Appl. 9(2), 195-205 (2007) 
24. Li, S, Stević, S: Generalized composition operators on Zygmund spaces and Bloch type spaces. J. Math. Anal. Appl. $338,1282-1295(2008)$

25. Li, S, Stević, S: Products of Volterra type operator and composition operator from $H^{\infty}$ and Bloch spaces to Zygmund spaces. J. Math. Anal. Appl. 345, 40-52 (2008)

26. Li, S, Stević, S: Products of composition and integral type operators from $H^{\infty}$ to the Bloch space. Complex Var. Elliptic Equ. 53(5), 463-474 (2008)

27. Li, S, Stević, S: Composition followed by differentiation between Bergman spaces and Bloch type spaces. J. Appl. Funct. Anal. 3(3), 333-340 (2008)

28. Li, S, Stević, S: Composition followed by differentiation between $H^{\infty}$ and $\alpha$-Bloch spaces. Houst. J. Math. 35, 327-340 (2009)

29. Liu, Y, Yu, Y: Weighted differentiation composition operators from mixed-norm to Zygmund spaces. Numer. Funct. Anal. Optim. 31, 936-954 (2010)

30. Liu, X, Yu, Y: The product of differentiation operator and multiplication operator from $H^{\infty}$ to Zygmund spaces. J. Xuzhou Norm. Univ. Nat. Sci. Ed. 29, 37-39 (2011)

31. Liu, Y, Yu, Y: Composition followed by differentiation between $H^{\infty}$ and Zygmund spaces. Complex Anal. Oper. Theory 6, 121-137 (2012)

32. Stević, S: Generalized composition operators from logarithmic Bloch spaces to mixed-norm spaces. Util. Math. 77, 167-172 (2008)

33. Stević, S: Norm and essential norm of composition followed by differentiation from $\alpha$-Bloch spaces to $H_{\mu}^{\infty}$. Appl. Math. Comput. 207(1), 225-229 (2009)

34. Stević, S: Products of composition and differentiation operators on the weighted Bergman space. Bull. Belg. Math. Soc. Simon Stevin 16(4), 623-635 (2009)

35. Stević, S: Weighted differentiation composition operators from mixed-norm spaces to weighted-type spaces. Appl. Math. Comput. 211, 222-233 (2009)

36. Stević, S: Composition followed by differentiation from $H^{\infty}$ and the Bloch space to $n$th weighted-type spaces on the unit disk. Appl. Math. Comput. 216(12), 3450-3458 (2010)

37. Stević, S: Weighted differentiation composition operators from $H^{\infty}$ and Bloch spaces to $n$th weighted-type spaces on the unit disk. Appl. Math. Comput. 216(12), 3634-3641 (2010)

38. Stević, S: Weighted differentiation composition operators from the mixed-norm space to the $n$th weighted-type space on the unit disk. Abstr. Appl. Anal. 2010, Article ID 246287 (2010)

39. Ohno, S: Products of composition and differentiation between Hardy spaces. Bull. Aust. Math. Soc. 73(2), 235-243 (2006)

40. Hibschweiler, RA, Portnoy, N: Composition followed by differentiation between Bergman and Hardy spaces. Rocky Mt. J. Math. 35(3), 843-855 (2005)

41. Li, S, Stević, S: Composition followed by differentiation from mixed-norm spaces to $\alpha$-Bloch spaces. Sb. Math. 199(12), 1847-1857 (2008)

42. Yang, W: Products of composition and differentiation operators from $Q_{K}(p, q)$ spaces to Bloch-type spaces. Abstr. Appl. Anal. 2009, Article ID 741920 (2009)

43. $Y u, Y, L i u, Y: T h e$ product of differentiation operator and multiplication operator from the mixed-norm to Bloch-type space. Acta Math. Sci. Ser. A Chin. Ed. 32, 68-79 (2012) (Chinese)

44. Zhu, X: Products of differentiation, composition and multiplication from Bergman type spaces to Bers type spaces. Integral Transforms Spec. Funct. 18, 223-231 (2007)

45. Kumar, S, Singh, KJ: Weighted composition operators on weighted Bergman spaces. Extr. Math. 22(3), 245-256 (2007)

46. Čučkovič, Z, Zhao, R: Weighted composition operators between different weighted Bergman spaces and different Hardy spaces. III. J. Math. 51(2), 479-498 (2007)

47. Liang, Y, Zhou, Z: Essential norm of the product of differentiation and composition operators between Bloch-type spaces. Arch. Math. 100(4), 347-360 (2013)

48. Liang, Y, Zhou, Z: New estimate of essential norm of composition followed by differentiation between Bloch-type spaces. Banach J. Math. Anal. 8(1), 118-137 (2014)

49. Ye, S: Norm and essential norm of composition followed by differentiation from logarithmic Bloch spaces to $H_{\mu}^{\infty}$. Abstr. Appl. Anal. 2014, Article ID 725145 (2014)

50. Hyvärinen, $\mathrm{O}$, Nieminen, I: Weighted composition followed by differentiation between Bloch-type spaces. Rev. Mat. Complut. 27, 641-656 (2014)

51. Liang, Y, Zhou, Z: Product of extended Cesàro operator and composition operator from Lipschitz space to $F(p, q, s)$ space on the unit ball. Abstr. Appl. Anal. 2011, Article ID 152635 (2011)

52. Liu, Y, Zhou, J: On an operator $M_{u} \mathcal{R}$ from mixed norm spaces to Zygmund-type spaces on the unit ball. Complex Anal. Oper. Theory 7, 593-606 (2013)

53. Liu, Y, Yu, Y: Products of composition, multiplication and radial derivative operators from logarithmic Bloch spaces to weighted-type spaces on the unit ball. J. Math. Anal. Appl. 423(1), 76-93 (2015)

54. Stević, S: On a product-type operator from Bloch spaces to weighted-type spaces on the unit ball. Appl. Math. Comput. 217(12), 5930-5935 (2011)

55. Stević, S: Weighted radial operator from the mixed-norm space to the $n$th weighted-type space on the unit ball. Appl. Math. Comput. 218, 9241-9247 (2012)

56. Krantz, S, Stević, S: On the iterated logarithmic Bloch space on the unit ball. Nonlinear Anal. TMA 71(5-6), 1772-1795 (2009)

57. Stević, S: Norms of some operators from Bergman spaces to weighted and Bloch-type space. Util. Math. 76, 59-64 (2008)

58. Stević, S: On a new operator from the logarithmic Bloch space to the Bloch-type space on the unit ball. Appl. Math. Comput. 206(1), 313-320 (2008)

59. Stević, S: On a new integral-type operator from the Bloch space to Bloch-type spaces on the unit ball. J. Math. Anal. Appl. 354(2), 426-434 (2009)

60. Stević, S: On an integral operator between Bloch-type spaces on the unit ball. Bull. Sci. Math. 134(4), 329-339 (2010) 
61. Zhou, J, Liu, Y: Products of radial derivative and multiplication operators from $F(p, q, s)$ to weighted-type spaces on the unit ball. Taiwan. J. Math. 17(1), 161-178 (2013)

62. Zhou, J, Liu, Y: Products of radial derivative and multiplication operator between mixed norm spaces and Zygmund-type spaces on the unit ball. Math. Inequal. Appl. 17, 349-366 (2014)

63. Cowen, C, MacCluer, B: Composition Operators on Spaces of Analytic Functions. Studies in Advanced Mathematics. CRC Press, Boca Raton (1995)

64. Tjani, M: Compact composition operators on some Moebius invariant Banach spaces. Dissertation, Michigan State University (1996)

65. Madigan, K, Matheson, A: Compact composition operator on the Bloch space. Trans. Am. Math. Soc. 347, 2679-2687 (1995)

66. Montes-Rodriguez, A: Weighted composition operators on weighted Banach spaces of analytic functions. J. Lond. Math. Soc. 61, 872-884 (2000)

Submit your manuscript to a SpringerOpen ${ }^{\odot}$ journal and benefit from:

- Convenient online submission

Rigorous peer review

- Immediate publication on acceptance

- Open access: articles freely available online

- High visibility within the field

- Retaining the copyright to your article 